\title{
Joined up mouth care for people with type 2 diabetes
}

People with type 2 diabetes are to get joined up care when receiving dental treatment thanks to new standards set by NHS England and NHS Improvement.

This new standard supports the ambition in the NHS Long Term Plan to move towards

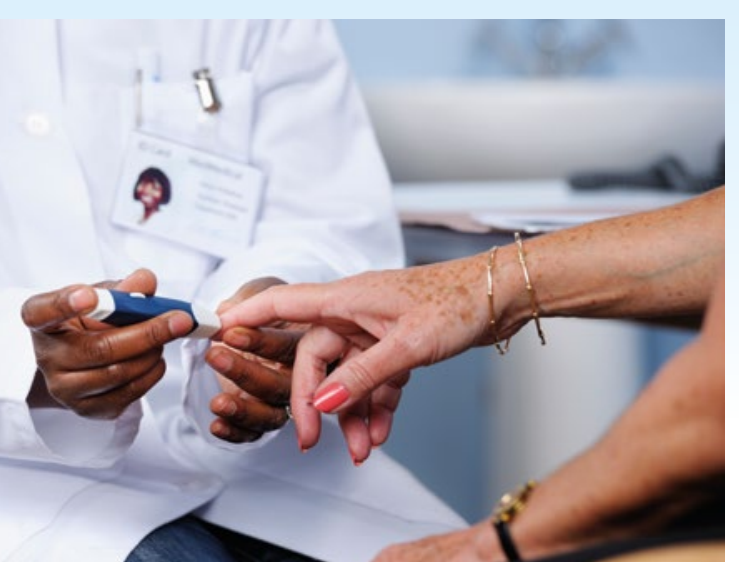

a care service model in which patients will get improved support through joined up care in a timely manner.

Evidence shows a two-way relationship between type 2 diabetes and periodontitis. This means people with type 2 diabetes are at greater risk of developing periodontitis, and people with periodontitis are at greater risk of developing type 2 diabetes.

With effective management of oral health and treatment of periodontitis, people can reduce their $\mathrm{HbA1C}$ levels which is crucial to managing type 2 diabetes.

This Standard sets out the type of a care a person with diabetes can receive from the dental team: $\rightarrow$ A full dental examination

$\rightarrow$ Assessment of their gum health

$\rightarrow$ Expert advice on maintaining excellent oral health.

Sara Hurley, Chief Dental Officer for England said: 'The consequences of periodontitis disease and type 2 diabetes can be devastating, the new standards will ensure people can take better control over their own health. With evidence now showing the clear link between periodontitis and type 2 diabetes, it is appropriate for NHS England and NHS Improvement to be issuing new standards to ensure people receive the best care possible.'

The full standard is available at: https:// www.england.nhs.uk/primary-care/ dentistry/dentistry-publications/\#standards

\section{New guidelines to curb remote prescribing of dermal fillers 'don't go far enough'}

The British Association of Oral and Maxillofacial Surgeons (BAOMS) has welcomed the publication of new guidance from the Joint Council for Cosmetic Practitioners (JCCP) on the remote prescription of medication for non-surgical cosmetic treatments such as dermal fillers, but said it did not go far enough.

The NHS faces increasing costs to treat the high street procedures that go wrong. But, without NHS coding for non-surgical treatment problems the scale of emergency treatment and corrective surgery remains unknown.

Caroline Mills, British Association of Oral and Maxillofacial Surgeons (BAOMS) Lead on Aesthetic/Cosmetic Surgery said: 'While BAOMS agrees with the introduction of guidelines for remote prescription of dermal fillers and other medications for high street beauty salon practitioners, we want to see regulation of the industry in line with European Union (EU) rules.

'The new JCCP guidance to curb remote prescribing will provide some protection for patients, but it does not go far enough. In the EU practitioners have to have a medical licence to inject fillers, and we need similar regulation in the UK.

'Even with the new guidelines where the prescriber will assess the patient's suitability for treatment, there is still the risk of serious medical complications because the regulations allow non-medical staff to give non-surgical injectables.
Dr Mills explaied that patients can suffer vascular occlusion, possibly leading to blindness, or severe allergic reactions both of which require emergency medical treatment.

'It's recognising and managing these problems that is so important and where patient safety may be compromised', Mills added. 'We need regulation in the UK to protect patients properly'

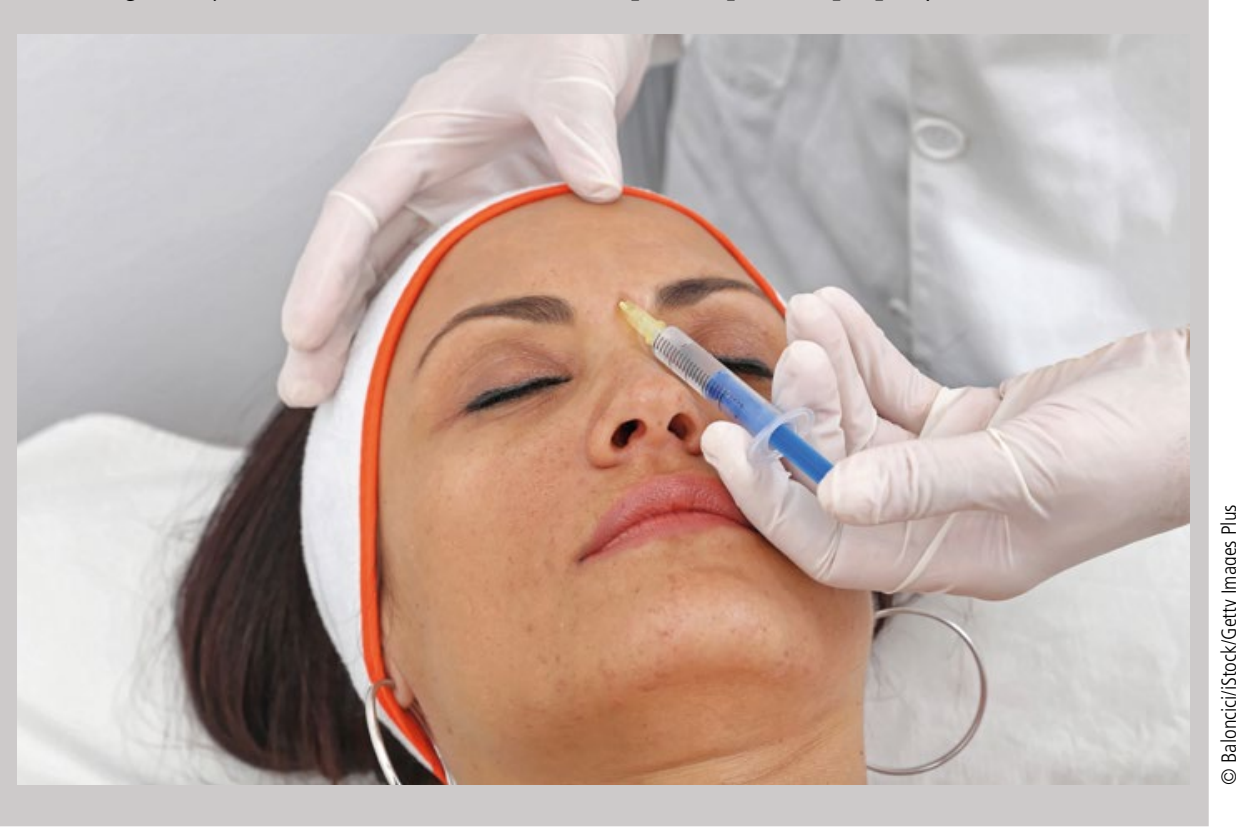

